\title{
Parathyroid-related protein plays a critical role in bone invasion by oral squamous cell carcinoma
}

\author{
YUKI TAKAYAMA ${ }^{1,2}$, TAISUKE MORI ${ }^{2}$, TAKESHI NOMURA ${ }^{1}$, \\ TAKAHIKO SHIBAHARA $^{1}$ and MICHIIE SAKAMOTO ${ }^{2}$
}

\author{
${ }^{1}$ Department of Oral and Maxillofacial Surgery, Tokyo Dental College, 1-2-2 Masago, Mihama-ku, Chiba 261-8502; \\ ${ }^{2}$ Department of Pathology, School of Medicine, Keio University, 35 Shinanomachi, Shinjuku-ku, Tokyo 160-8582, Japan
}

Received August 20, 2009; Accepted October 14, 2009

DOI: 10.3892/ijo_00000623

\begin{abstract}
Bone invasion is a critical prognostic factor for patients with oral squamous cell carcinoma (OSCC). We established an orthotropic implantation model using the murine OSCC cell line, SCCVII, showing direct invasion of the mandible by OSCC. Using this model, we examined the molecular mechanisms of bone invasion and the role of parathyroid-related protein (PTHrP). We established PTHrP, stable, knock-down SCCVII cells. Knock-down of PTHrP caused decreased osteoclast formation in vitro relative to expression levels of PTHrP. In vivo models showed dramatic suppression of bone invasion in PTHrP knock-down cells, and the degree of suppression was more pronounced than the level of PTHrP knock-down. We looked at an additive role of transforming growth factor- $\beta$ (TGF- $\beta$ ) in PTHrP-mediated bone invasion. TGF- 3 induced mRNA expression of PTHrP, showed no inhibitory effect on SCCVII cell proliferation, and caused epithelial mesenchymal trans-differentiation such as changes in the cells. Sections of resected mandibles from patients with invasive OSCC showed a great number of osteoclasts at bone invasion sites, strong expression of PTHrP, and decreased expression of E-cadherin in the tumour cells. Cancer-derived PTHrP appears to play a critical role in bone invasion by OSCC, mediated by osteoclasts. Moreover, TGF- $\beta$ appears to act synergistically to accelerate mandibular bone invasion.
\end{abstract}

\section{Introduction}

Squamous cell carcinomas account for more than $90 \%$ of malignant tumours of the oral cavity $(1,2)$. Advanced oral squamous cell carcinoma (OSCC) frequently invades adjacent bones, causing bone destruction, pain, hypercalcaemia, fractures and nerve paralysis, which become major clinical

Correspondence to: Dr Michiie Sakamoto, Department of Pathology, School of Medicine, Keio University, 35 Shinanomachi, Shinjuku-ku, Tokyo 160-8582, Japan

E-mail: msakamot@sc.itc.keio.ac.jp

Key words: oral squamous cell carcinoma, bone invasion, PTHrP, animal model issues (3-5). Patients with OSCC and associated bone invasion require bone resection, which has a major influence on their quality of life and is a critical factor in their functional outcome. However, the mechanism by which OSCC invades bone and the means to prevent this invasion, remains unresolved. An understanding of the molecular mechanism of bone invasion associated with OSCC is thus urgently needed.

Animal models are essential for studying the mechanisms of mandibular invasion by OSCC. Our previous attempt to establish an animal model using a human OSCC cell line was unsuccessful, however, we have now established a murine mandibular bone invasion model using the murine OSCC cell line, SCCVII (6). The growth of SCCVII cells is unaffected by the immune system. SCCVII cells directly invade the mandible when injected into the masseter muscle of $\mathrm{C} 3 \mathrm{H} / \mathrm{HeN}$ mice $(7,8)$. Several human cancers such as breast cancer $(9,10)$, prostate cancer $(11,12)$, and myeloma $(13,14)$ metastasise to bone. Direct bone invasion and metastasis by cancer cells is reportedly mediated by osteoclasts rather than being directly caused by cancer cells (3). Parathyroid hormone-related protein (PTHrP) is reported to be responsible for osteoclast-stimulating activity and bone destruction in breast carcinoma $(15,16)$. The PTHrP secreted from tumour cells enhances osteoclastogenesis by inducing the expression of receptor activator of nuclear factor $\kappa \mathrm{B}$ ligand (RANKL) in marrow stroma cells $(17,18)$. RANKL stimulates RANK (receptor activator for nuclear factor $\kappa \mathrm{B}$ ), which is expressed on the surface of osteoclast progenitor cells, and the RANK signal promotes osteoclastogenesis. The process of bone resorption releases transforming growth factor- $\beta$ (TGF- $\beta$ ) which increases the production of PTHrP by tumour cells, and the production of growth factors that increase tumour growth (19).

We previously analysed bone invasion patterns (20) and immunohistochemistry (21) using mandibular bone specimens obtained from patients with OSCC and mandibular invasion. These analyses demonstrated that the presence of osteoclast activation factors including PTHrP, tumour necrosis factor- $\alpha$ (TNF- $\alpha$ ), interleukin-6 (IL-6) and TGF- $\beta$ is important for this process (21). In particular, it was found that, in almost all patients with OSCC and bone invasion, the tumour stained positively for PTHrP.

Using our murine model we have examined the molecular mechanism of bone invasion in OSCC, focusing on the roles of PTHrP and TGF-ß. 


\section{Materials and methods}

Cell lines, murine bone invasion models and clinical samples. SCCVII cells were derived from a murine OSCC cell line (8). The SCCVII cells were cultured in Dulbecco's modified Eagle's medium (Sigma, St. Louis, MO, USA) containing 10\% fetal bovine serum, $100 \mu \mathrm{g} / \mathrm{ml}$ streptomycin and $100 \mu \mathrm{g} / \mathrm{ml}$ ampicillin. They were incubated at $37^{\circ} \mathrm{C}$ in a humidified atmosphere with $5 \% \mathrm{CO}_{2}$. NIH3T3 cells, derived from a cell line of murine fetal fibroblasts, were cultured under the same conditions and acted as a positive control.

Twenty-one male $\mathrm{C} 3 \mathrm{H} / \mathrm{HeN}$ mice, weighing about $20 \mathrm{~g}$ and between seven to nine weeks of age, were used. The bone invasion model was constructed as described previously (6). Animal experiments were conducted according to the Guidelines for the Treatment of Experimental Animals at Tokyo Dental College. The animals in the SCCVII transplant group were maintained for three weeks after injection with the cells. At the end of week three, all surviving mice were sacrificed.

We recruited 18 patients with mandibular gingival SCC. All patients were recruited from the Department of Oral and Maxillofacial Surgery at the Tokyo Dental College Chiba Hospital in the period between 2004 and 2007. All patients were treated with surgical excision of the primary lesion by mandibulectomy. Informed consent was obtained from each patient according to a protocol approved by the Ethics Review Board of Tokyo Dental College (approval \#A05-0460-1).

Real-time quantitative reverse transcription (RT)-PCR. Conditions for the real-time quantitative reverse transcription PCR (qRT-PCR) analysis have been described previously (22). The following primer sets were used: 5'-CAACTTGTTCAA GGGCGTTGTG-3' (forward), 5'- TGTGGCTCCCATAGCA ATGTCTAA-3' (reverse) PTHrP; 5'-TTGCAAGTTCCTGC CATCCTC-3' (forward), 5'-CACATTGTCCCGGGTATCA TCA-3' (reverse) glyceradehyde-3-phosphate dehydrogenase. The PTHrP mRNA expression levels were normalized with glyceradehyde-3-phosphate dehydrogenase. All primers were designed to target a coding region and to cross an exon junction. Real-time detection of the emission intensity of SYBR Green was performed using a Thermal Cycler Dice ${ }^{\mathrm{TM}}$ Real Time System and an SYBR ${ }^{\circledR}$ Premix Ex Taq ${ }^{\mathrm{TM}}$ kit (Perfect Real Time) (Takara Bio Inc., Shiga, Japan). Quantitative RT-PCR was performed at least three times, including a notemplate negative control.

Immunofluorescence and immunoblotting. Immunofluorescence and immunoblotting analyses were performed as described previously (22). Immunofluorescent analysis was repeated twice, and all samples were observed using a confocal laser-scanning microscope (LSM510, Carl Zeiss, Germany). A primary rabbit, PTHrP polyclonal antibody (Santa Cruz Biotechnology, CA, USA) was applied at a dilution of 1:200. A $\beta$-actin antibody (Sigma) was employed as a loading control.

Vector construction and retroviral infection. The destination vector, pDEST-CMSCVpuro, the retroviral short hairpin RNA (shRNA) expression vector, pDEST-CL-SI-MSCVpuro (formerly designated as pSI-CMSCVpuroDEST), and the entry vector, pENTR-H1R-stuffer, were constructed in accordance with previous reports (22). Two shRNA expression vectors for PTHrP, pSI-CMSCV puro-H1R-PTHrPshRNA\#1 and pSICMSCVpuro-H1R-PTHrPshRNA\#2, containing the 5'-GCT CAAACGCGCTGTGTCT-3' and 5'-GGAAACTGCACATG GGTCA-3' target sequences, respectively, were constructed. As a control (non-targeting sequence), the sequence 5'-TAAGG CTATGAAGAGATAC-3' was used in the shRNA expression vector $\mathrm{pSI}-\mathrm{CMSCV}$ puro-H1R-Control. The production of recombinant retroviruses was performed as described previously (22). The two series of PTHrP stable knock-down SCCVII cells and the control SCCVII cells were designated as shRNA\#1, shRNA\#2, and shControl, respectively.

Osteoclast formation assay. Mouse bone marrow cells $\left(1 \times 10^{6}\right)$ were isolated from 7 -week old $\mathrm{C} 3 \mathrm{H} / \mathrm{HeN}$ mice and co-cultured in 24-well plates with PTHrP knock-down SCCVII cells $\left(1 \times 10^{5}\right)$ in $0.5 \mathrm{ml}$ of $\alpha$ modified minimum essential medium (MEM) (Sigma) containing 10\% fetal bovine serum (FBS) in the presence of 1,25 dihydroxy vitamin $\mathrm{D}_{3}\left(10^{-8} \mathrm{M}\right)$ and dexamethasone $\left(10^{-7} \mathrm{M}\right)$. After 7 days, the cells were fixed and stained for tartrate-resistant acid phosphatase (TRAP). TRAP-positive, multinucleated cells, containing more than three nuclei, were counted as osteoclasts and counts were expressed as osteoclasts per well.

Micro-computed tomography, three-dimensional reconstruction and counting of osteoclasts. We implanted the SCCVII cells into three groups of 7 mice each (shControl, shRNA\#1, and shRNA\#2 groups). All mice were confirmed as showing tumour formation. Micro-computed tomography $(\mu-\mathrm{CT})$ scans of all mice heads were taken and reconstructed with a KMS-755 microtomographic system (Pony Co., Kashimura, Osaka, Japan) and a KMS755 microfocus CT Rebuilder (Version 1.00, Image Script Co., Ltd., Kashimura, Osaka, Japan). The photographs were reconstructed threedimensionally using a dedicated software package (TRI/3D Bon; R 2.1.16-S Ratoc, Tokyo, Japan). We also determined the tumour volume and bone invasion on $\mu$-CT sections.

Four-micron thick frontal sections were made through the centre of the mandibular tumours. Osteoclasts from the tumour/bone interface were assessed at the invasion sites using TRAP staining. The number of osteoclasts at the sites of bone invasion was assessed using an average of 20 sites per section.

The effect of TGF- $\beta$ on SCCVII cells in vitro. SCCVII cells were cultured with TGF- 3 (R\&D, MN, USA) in Opti-MEM medium (Gibco, NY, USA). SCCVII cell proliferation was analyzed at 24,48 , and $72 \mathrm{~h}$ by counting the number of cells using a blood cell counting chamber. The levels of expression of PTHrP, E-cadherin, vimentin, and snail1 in SCCVII cells after $72 \mathrm{~h}$ of culture with TGF- $3(5 \mathrm{ng} / \mathrm{ml})$ were analyzed by qRT-PCR. The following primer sets were used: 5'-TTGCA AGTTCCTGCCATCCTC-3' (forward), 5'-CACATTGTCCC GGGTATCATCA-3' (reverse) E-cadherin; 5'-AAAGCGTG GCTGCCAAGAAC-3' (forward), 5'-GTGACTGCACCTGT CTCCGGTA-3' (reverse) vimentin; 5'-GTGTCTGCACGAC CTGTGGAA-3' (forward), 5'-ACAGGCCTGGCACTGGTA TCTC-3' (reverse) snaill. 
A

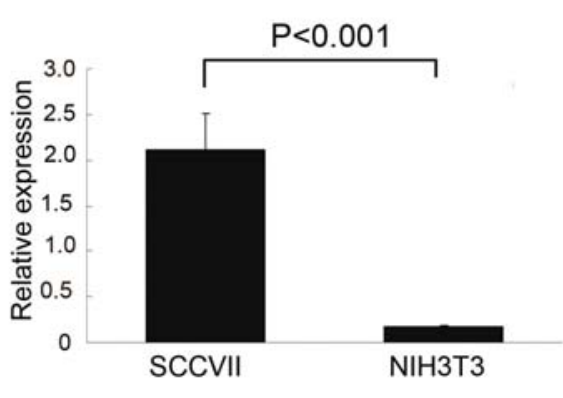

B

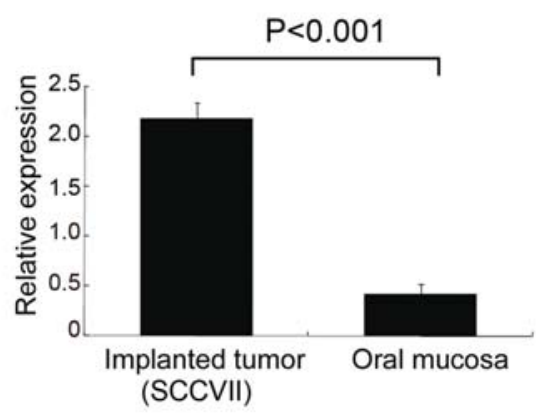

C

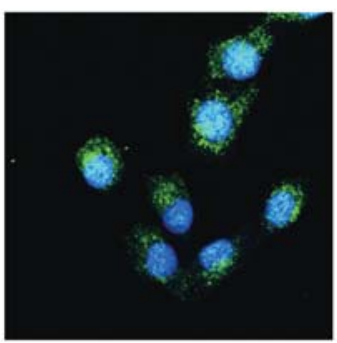

$\mathrm{D}$

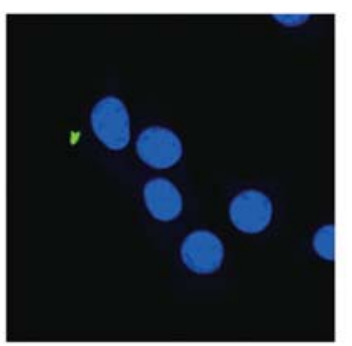

Figure 1. Parathyroid-related protein (PTHrP) is expressed at the mRNA and protein levels in SCCVII cells. (A) Expression of PTHrP in a mouse oral squamous cell carcinoma (OSCC) cell line, SCCVII cells, was higher than in the control cell line, NIH3T3, as determined by quantitative real-time PCR (qRT-PCR) analysis. The bars show the mean \pm SD. ${ }^{*} \mathrm{P}<0.001$. (B) The level of expression of PTHrP mRNA in implanted SCCVII cells was higher than that in the oral mucosa tissue of $\mathrm{C} 3 \mathrm{H} / \mathrm{HeN}$ mice (controls) as determined by qRT-PCR. The bars show the mean $\pm \mathrm{SD}$. ${ }^{*} \mathrm{P}<0.001$. (C) Immunofluorescent analysis of SCCVII cells with an anti-PTHrP antibody (green) and DNA stain DAPI (blue). The SCCVII cells stained positive for PTHrP in the cell cytoplasm. (D) Immunofluorescent analysis of SCCVII cells without anti-PTHrP antibody as a negative control.

Immunohistochemical staining of resected human mandibles. Resected tumour and mandibular blocks from patients with gingival SCC were fixed with $10 \%$ paraformaldehyde, decalcified in formic acid and embedded in paraffin. Sections $(5 \mu \mathrm{m})$ were stained with haematoxylin and eosin $(\mathrm{H} \& \mathrm{E})$. Immunohistochemical staining was performed using CD68 antibody (Dako, clone Kp-2, working dilution 1:200), PTHrP antibody (Calbiochem, working dilution 1:200) and Ecadherin antibody (NCL36B5, working dilution 1:100). TRAP staining is not suitable for decalcified specimens, therefore we used CD68 antibodies to detect osteoclasts. Specimens treated with PBS alone were used as negative controls. Specimens where staining in the cytoplasm was more pronounced than the negative controls were regarded as positive.

Statistical analysis. The Student's t-test was used to assess the statistical significance of the difference between means. A $\mathrm{P}<0.05$ was considered to be statistically significant.

\section{Results}

PTHrP over-expression in a mouse OSCC cell line (SCCVII). The level of expression of PTHrP in the cultured SCCVII cells was at least 10-fold higher than in the cultured PTHrP-positive NIH3T3 cells $(\mathrm{P}<0.001)$ (Fig. 1A). The level of expression of PTHrP in the implanted SCCVII tumour cells was 4-fold higher than that in the background oral mucosa tissues $(\mathrm{P}<0.001)$ (Fig. 1B). Immunofluorescence showed that the cytoplasm of the SCCVII cells stained positive for PTHrP
(Fig. 1C and D) confirming that SCCVII cells express high amounts of PTHrP.

Stable knock-down of PTHrP. Stable PTHrP knock-down cells, generated by RNA interference technology using retrovirus vectors, were used to evaluate the role of PTHrP in bone invasion. qRT-PCR and immunoblotting showed statistically significant reductions in the levels of PTHrP mRNA and protein in the two series of stable PTHrP knock-down SCCVII cells (shRNA\#1 and shRNA\#2) compared with the control cells (shControl) in vitro (shCont. vs shRNA\#1, $\mathrm{P}=0.04$; shCont. vs shRNA\#2, $\mathrm{P}=0.01$ ) (Fig. $2 \mathrm{~A}$ and $\mathrm{B}$ ). The reduction in mRNA PTHrP levels was more significant in the shRNA\#2 group. These results suggested that a stable, PTHrP knockdown was successfully generated in SCCVII cells. These cell lines were used for further analysis.

Reduction in osteoclast formation in PTHrP knock-down cells. To determine the effect of PTHrP suppression, we analyzed osteoclast formation in transfected SCCVII cells, shControl, shRNA\#1, and shRNA\#2. Both shRNA\#1 and shRNA\#2 showed significantly fewer osteoclasts than the shControl cells ( shRNA\#1, $\mathrm{P}=0.02$; shRNA\#2, $\mathrm{P}=0.002$ ) (Fig. 2C). There were fewer osteoclasts in the shRNA\#2 than in the shRNA\#1 cells. This suggested that the stable knock-down of PTHrP caused a decrease in the number of osteoclasts relative to the expression levels of PTHrP.

Orthotopic murine model of bone invasion. The effect of the PTHrP knock-down was analyzed using the murine 


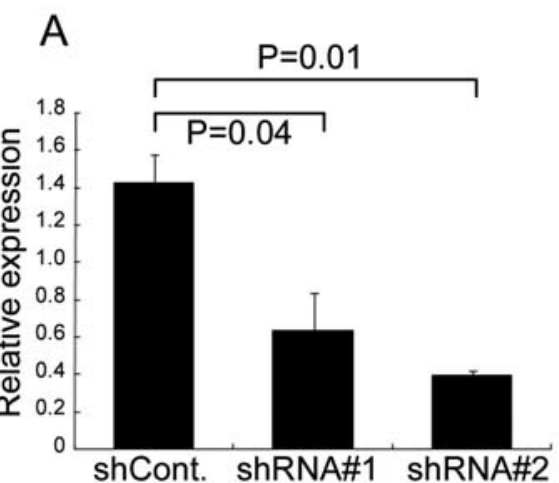

\section{C}

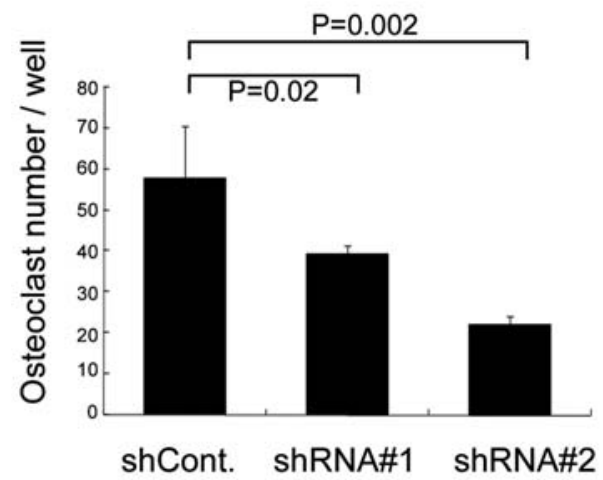

model in vivo. We analyzed bone invasion in each mouse group using three-dimensional reconstructions of the skull created from $\mu$-CT sectioning as macroscopic observations, and $\mathrm{H} \& \mathrm{E}$ and TRAP staining as microscopic observations (Fig. 3A-L).

Expression of PTHrP mRNA in the implanted tumours was decreased in the shRNA\#1 and shRNA\#2 groups compared with the shControl (Fig. 3M). These results confirmed that the implanted SCCVII cells continued to display the effects of the PTHrP knock-down. Samples from the shControl group in the upper panel (Fig. 3A-D) show severe destruction of the mandible (Fig. 3A arrow) and zygoma (Fig. 3B arrowhead). In fact, the mandible and zygoma have nearly disappeared. Samples from the shRNA\#1 group (Fig. 3E-H) and the shRNA\#2 group (Fig. 3I-L) showed deformation of the mandible and zygoma without destruction. The mandibles were asymmetric and the zygomata were bowed. We determined bone resorption from $\mu$-CT sections and computer modelling. The mean bone resorption volumes in the shRNA\#1 (mean volume: $1.089 \mathrm{~mm}^{3}$ ) and shRNA\#2 groups (mean volume: $0.632 \mathrm{~mm}^{3}$ ) were about 5 -fold lower than that in the shControl group (mean volume: $4.601 \mathrm{~mm}^{3}$ ) (Fig. $\left.3 \mathrm{~N}\right)$. H\&E staining (x40) of the shControl group specimen showed funicular invasion and a serrated pattern of bone resorption (Fig. 3C). H\&E staining ( $x 40)$ of the shRNA\#1 and shRNA\#2 group specimens did not show clear bone invasion (Fig. 3G and K). After TRAP staining (x200), osteoclasts were easily identified. Osteoclasts of the shControl group were found at the bone invasion site (Fig. 3D). Fewer osteoclasts were found outside the bone invasion site (Fig. $3 \mathrm{H}$ and $\mathrm{L}$ ). The number of osteoclasts in the shControl group was $\sim 3$-fold greater than the
B

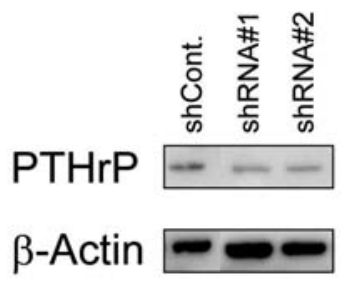

Figure 2. Stable parathyroid-related protein (PTHrP) knock-down in oral squamous cell carcinoma (OSCC) cells (SCCVII) using shRNA retrovirus vectors. (A and B) The level of expression of PTHrP was analyzed in a stable PTHrP knock-down mouse OSCC cell line (SCCVII cells) by quantitative real-time PCR (qRT-PCR) and immunoblotting analyses (using ß-actin, as a positive control). The results confirmed substantial reduction in PTHrP expression in the knock-down SCCVII cells (shRNA\#1 and shRNA\#2) compared to the shControl. (C) Osteoclast formation was reduced by PTHrP knock-down in SCCVII cells. Mouse bone marrow cells $\left(1 \times 10^{6}\right)$ were cocultured with SCCVII cells ( $1 \times 10^{4}$ cells) in 24-well plates. After 7 days the cells were fixed and stained for tartrate-resistant acid phosphatase (TRAP). The data show the number of osteoclasts per culture well. Similar results were obtained in three independent experiments.

number in the shRNA\#1 and shRNA\#2 groups (Fig. 3O). The number of osteoclasts followed a similar pattern to PTHrP expression. The mean tumour volume of the shControl group (mean volume $474.3 \mathrm{~mm}^{3}$ ) was larger than that of the shRNA\#2 group (mean volume $332.8 \mathrm{~mm}^{3} ; \mathrm{P}=0.03$ ), however, the difference between the shControl and shRNA\#1 groups was not statistically significant (mean volume $374.6 \mathrm{~mm}^{3}$; $\mathrm{P}=0.11$ ) (Fig. 3P).

The effects of TGF- $\beta$ on SCCVII cells in vitro. We evaluated the effects of TGF- $\beta$ on SCCVII cells in vitro. Initially, we confirmed the concentration of TGF- $B$, as previously recommended (23), and categorized the levels of TGF- $\beta$ into graduated concentrations $(0.5,1,5,10$, and $50 \mathrm{ng} / \mathrm{ml})$. PTHrP expression increased with increasing levels of TGF- $\beta$ in the concentration range $0.5-5 \mathrm{ng} / \mathrm{ml}$ (data not shown). The level of expression of PTHrP in the cells with TGF- $\beta(5 \mathrm{ng} / \mathrm{ml})$ was $13 \%$ higher than that in those cells without TGF- $\beta(\mathrm{P}=0.01)$ (Fig. 4A). We then confirmed the growth of SCCVII cells cultured with TGF- $\beta$. TGF- $\beta$ did not inhibit SCCVII cell proliferation in culture at any time-point $(24,48$, and $72 \mathrm{~h}$ ) (Fig. 4B). Phase-contrast images were taken after $72 \mathrm{~h}$ of culture with TGF- $\beta$. The SCCVII cells became spindle-shaped following TGF- $\beta$ treatment (Fig. $4 \mathrm{C}$ ). TGF- $\beta$ is related to epithelial mesenchymal transdifferentiation (EMT), so we measured the levels of the genes, E-cadherin, vimentin, and snail1. The level of expression of E-cadherin in the presence of TGF- $\beta$ was less than that observed without TGF- $\beta$ (Fig. 4D). The level of expression of vimentin and snaill in the presence of TGF- $\beta$ was greater than that observed without TGF- $\beta$. 
Side view
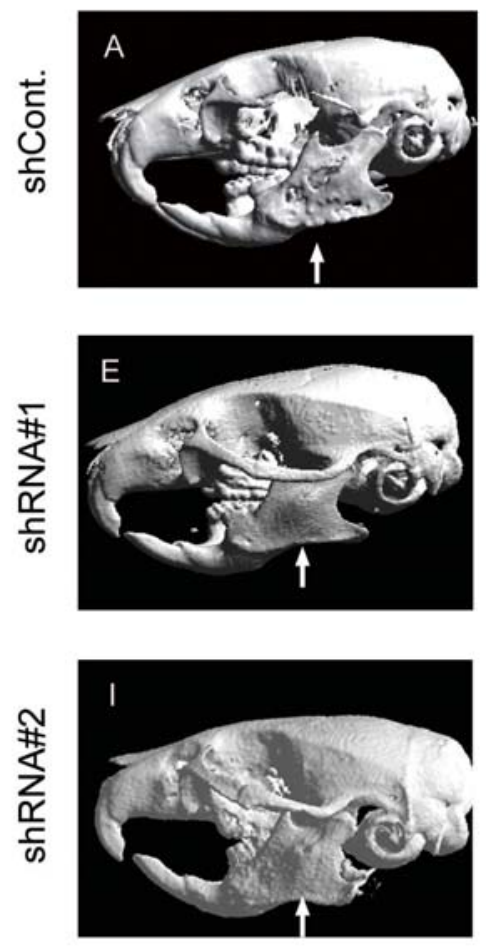

Top view
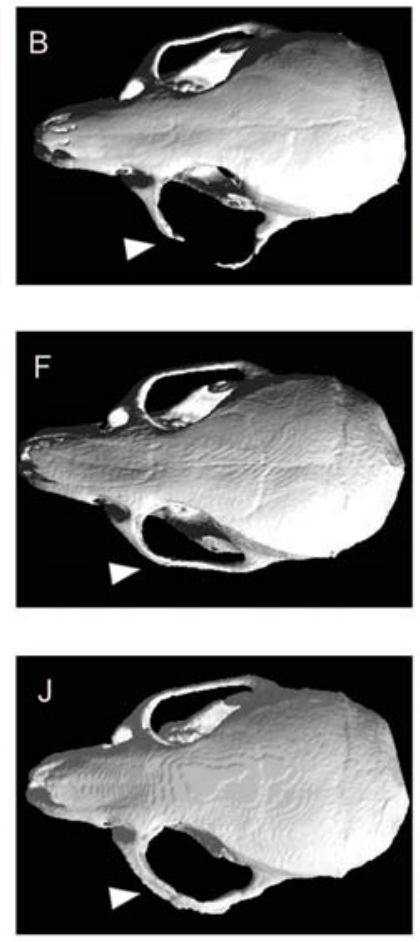

$\mathrm{H}-\mathrm{E}(\times 40)$
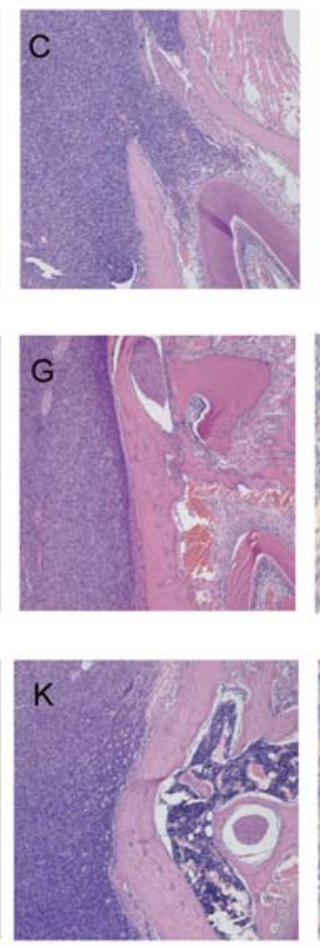

TRAP $(\times 200)$
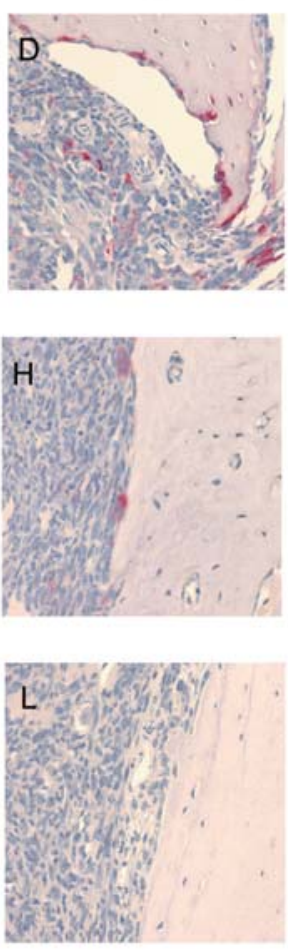

M
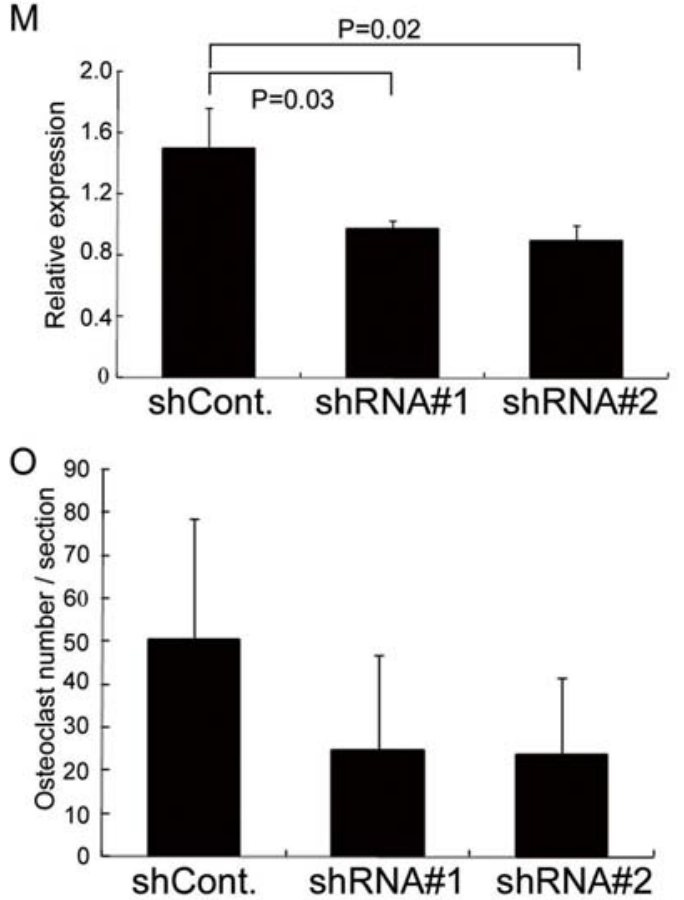

$\mathrm{N}$

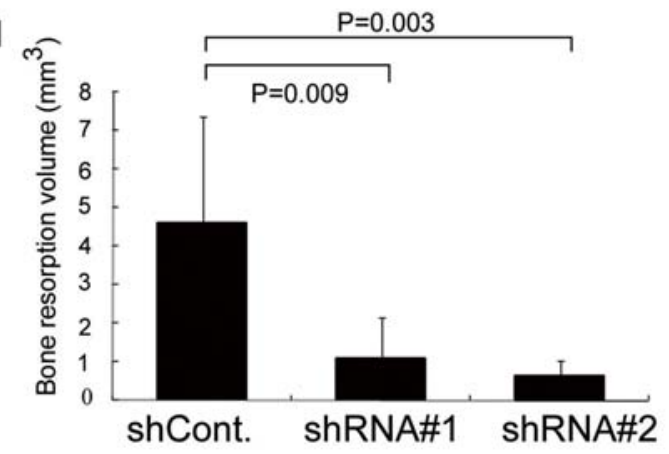

$\mathrm{P}$

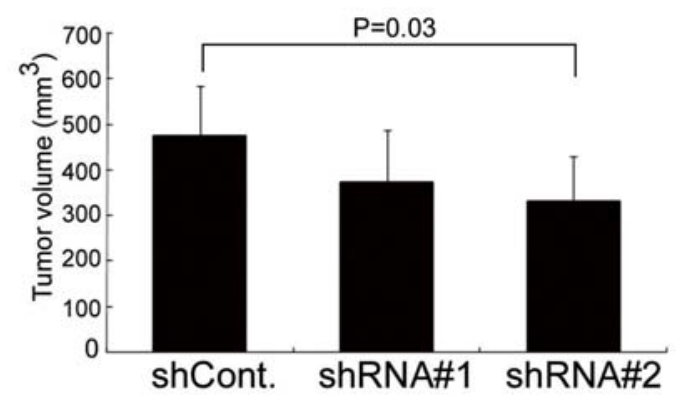

Figure 3. Animal model of bone invasion. (A-L) These figures show macroscopic findings from a three-dimensional reconstruction of the skull and microscopic findings from H\&E and tartrate-resistant acid phosphatase (TRAP) staining. (A, B, E, F, I and J) The side views (A, E and I) and top views (B, F and J) of the skull are shown in the macroscopic figures. Arrows show the mandible in the side view, and arrowheads showed the zygoma in the top view. (A and B) Samples from the shControl group (mice implanted with mouse oral squamous cell carcinoma cells - SCCVII cells) in the upper panel show severe bone resorption in the mandible and zygoma. Rough surfaces were observed in the mandible region, and unconnected bone was seen in the zygoma. (E, F, I and J) Samples from the parathyroid-related protein (PTHrP) knock-down SCCVII cells (shRNA\#1 group in the middle panel and (I and J) the shRNA\#2 group in the lower panel) display no significant change in either the mandible or zygoma. (C and D) H\&E staining ( $\mathrm{x} 40$ ) of the shControl group specimen showed marked bone resorption as indicated by the positive red colored osteoclasts in (D) TRAP staining (x200). (G, H, K and L) H\&E staining (x40) of the shRNA\#1 and shRNA\#2 group specimens showed no bone resorption in the boundary area as indicated by fewer or almost no positive red colored osteoclasts in (H and L) TRAP staining (x200). (M) The PTHrP mRNA expression levels were decreased in the shRNA\#1 and shRNA\#2 groups compared to the shControl. $(\mathrm{N})$ The bone resorption volume in the shControl group was larger than that in the shRNA\#1 and shRNA\#2 groups. (O) Osteoclast counts in the shControl group were larger than those in the shRNA\#1 and shRNA\#2 groups. (P) The tumour volume of the shControl was larger than that of the shRNA\#1 and shRNA\#2 groups. 
A

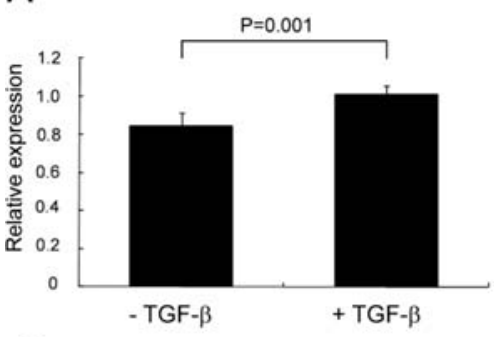

B

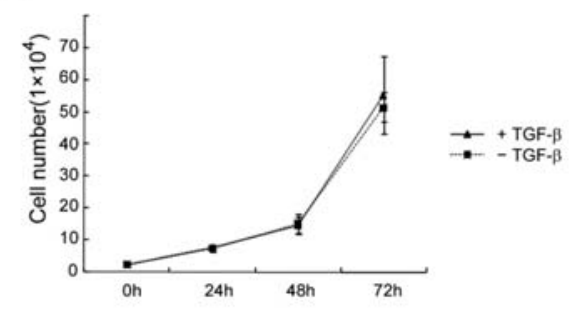

C

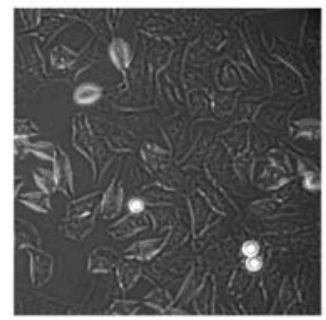

- TGF- $\beta$

D

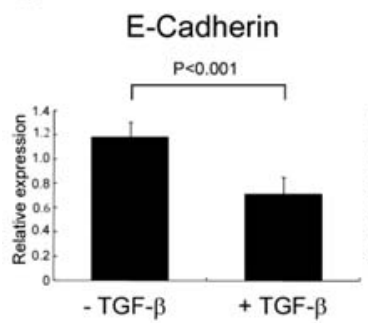

D

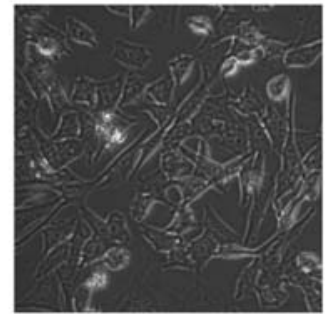

+ TGF- $\beta$

Figure 4. Effect of transforming growth factor- $\beta$ (TGF- $\beta$ ) in vitro. (A) TGF- $\beta$ induces parathyroid-related protein (PTHrP) mRNA levels. (B) SCCVII cells were treated with TGF- $\beta$. There were no significant changes in cell division. (C) A phase-contrast picture was taken after 72 h culture with TGF- $\beta$. The SCCVII cells were spindle-shaped following treatment with TGF- $\beta$. (D) The mRNA levels of epithelial mesenchymal transdifferentiation-related marker genes (E-cadherin, vimentin, and snail1) changed.
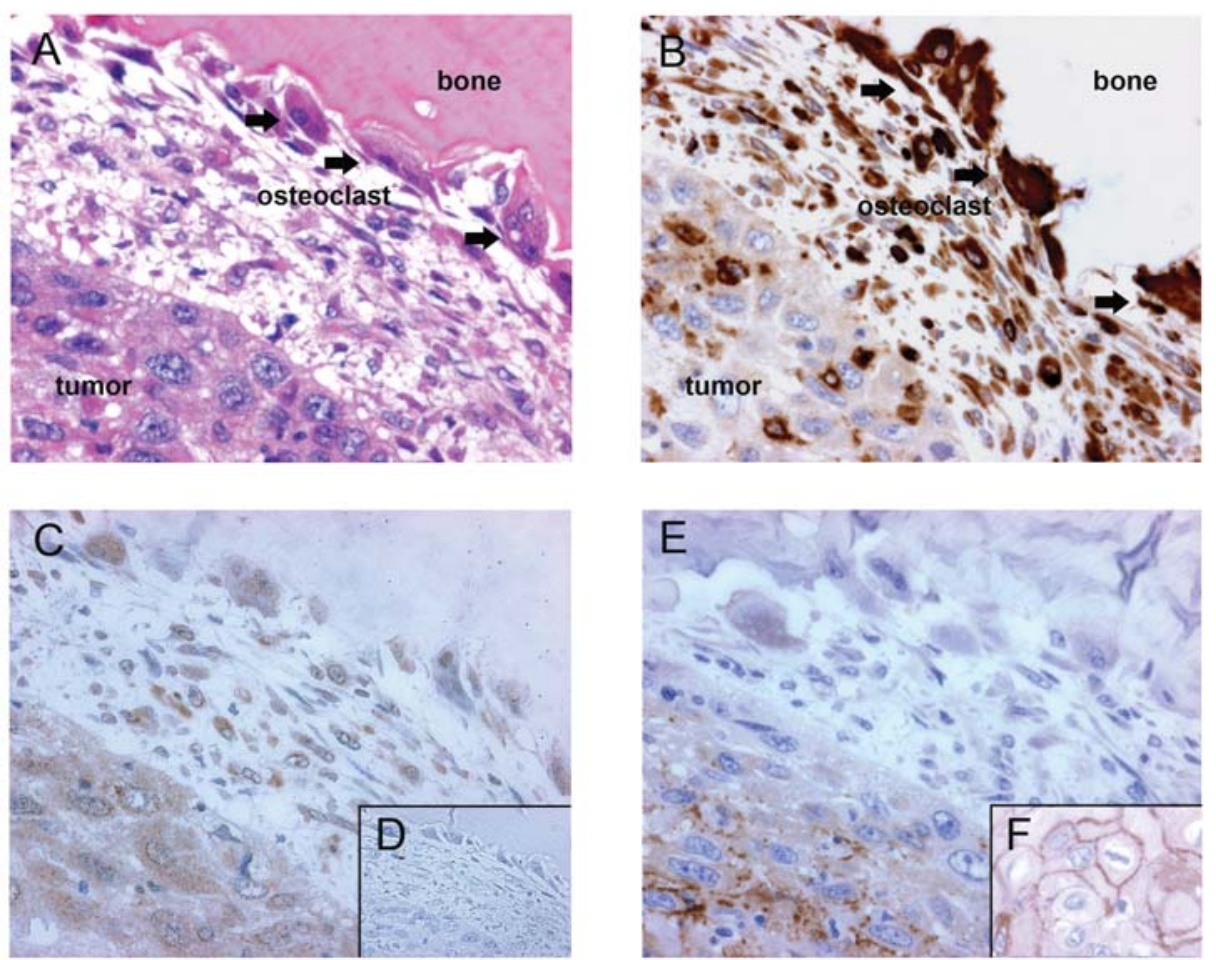

Figure 5. Oral squamous cell carcinoma (OSCC) invasion of the mandible and immunohistochemical staining. Histopathological findings of bone invasion in a surgical specimen. The tumour and bone boundary areas are shown. (A) H\&E; and (B) CD68 staining. Large numbers of osteoclasts (arrowheads) and macrophages were observed on the bone resorption surface. (C) Tumour cell cytoplasm stained positive for parathyroid-related protein. (D) Negative control. (E) The staining of E-cadherin in the tumour margin was weak in comparison with that in the central lesion (F). 
Immunohistochemical staining of OSCC in sections from patients with mandibular bone invasion. $\mathrm{H} \& \mathrm{E}$ staining showed a serrated pattern of bone invasion (Fig. 5A). Tumour cells showed no direct contact with osteoclasts or the mandible itself. Large numbers of monocytes, macrophages and osteoclasts were observed between the tumour and the bone at the bone invasion sites (Fig. 5B). Tumour cell cytoplasm stained positive for PTHrP (Fig. 5C), whereas the negative control, without PTHrP antibody, showed no staining (Fig. 5D). When staining the clinical specimens for E-cadherin, staining was weak at the bone resorption sites (Fig. 5E), but at the centre of the tumour, strong E-cadherin staining was observed around the cell membrane in a punctate pattern (Fig. 5F).

\section{Discussion}

PTHrP is a major mediator of osteoclast activity (24) and clinical analyses have suggested that osteoclast activation factors, including PTHrP, are highly expressed in patients with OSCC with bone invasion (21).

We firstly evaluated levels of PTHrP expression in mouse OSCC cell lines (SCCVII cells) with the ability to invade bone. High levels of expression of PTHrP mRNA and protein were observed in these SCCVII cells, as is seen in patients with OSCC with bone invasion (21). We generated stable PTHrP knock-down cells by RNA interference technology using retroviral vectors in order to evaluate the role of PTHrP in bone invasion. An in vitro osteoclast formation assay showed that both the shRNA\#1 and shRNA\#2 cells developed fewer osteoclasts than the shControl. This indicated that the stable PTHrP knock-down of SCCVII cells inhibited osteoclastogenesis in vitro. Using these cells we further verified the in vivo effect of stable PTHrP knock-down. We showed that the PTHrP knock-down inhibited bone invasion, and that the number of osteoclasts was associated with the level of PTHrP expression in SCCVII cells. The tumour volumes of the PTHrP knock-down SCCVII cells tended to be small, although the data did not show a significant statistical correlation between tumour volume and bone invasion. The mean bone resorption volume of the PTHrP knock-down SCCVII cells was significantly smaller than controls, implying that osteoclast activity is more important for active bone invasion than tumour volume.

In our in vivo murine model, the level of PTHrP expression in the shRNA\#1 and shRNA\#2 groups was $40 \%$ less than that of the shControl, while the bone resorption volumes of the ShRNA\#1 and shRNA\#2 groups were 75\% less than that of the shControl. The murine model showed that bone invasion was suppressed, and the suppression of the bone resorption was more pronounced than the PTHrP knockdown levels. These findings led to the hypothesis that microenvironmental factors have additive roles in PTHrP-mediated bone OSCC invasion. TGF- $\beta$ acts synergistically with PTHrP expression in breast cancer (19). TGF- $\beta$ is stored in bone and is released through bone resorption. Released TGF- $\beta$ increases the production of PTHrP by tumour cells as well as the production of growth factors that increase tumour growth (25). We verified the effects of TGF- $\beta$ in OSCC and, as expected, TGF- $\beta$ induced PTHrP mRNA expression. In addition, TGF- $\beta$ did not inhibit the growth of SCCVII cells but did induce
EMT-like changes. EMT in vitro is characterized by the reversible conversion of polarized epithelial cells into highly motile fibroblastoid cells $(26,27)$. Members of the TGF- $\beta$ family can initiate and maintain EMT $(28,29)$. EMT is associated with tumour invasiveness and intravasations and extravasations of metastatic cells (28).

The level of expression of EMT-associated genes such as E-cadherin, vimentin, and snaill were changed significantly in the presence of TGF- $\beta$, indicating that TGF- $\beta$ causes the EMT-like changes seen in the SCCVII cells. We considered that PTHrP-induced bone invasion caused TGF- $\beta$ release, PTHrP up-regulation and EMT of the cancer cells, which further accelerated bone invasion. These findings suggested that PTHrP acted synergistically with TGF- $\beta$ to accelerate mandibular bone invasion. Our analysis of patients with OSCC and bone invasion showed a great number of osteoclasts at the bone invasion sites, strong expression of PTHrP and reduced expression of E-cadherin in tumour cells. Findings from the murine model and clinical studies suggest that PTHrP from tumour cells activates osteoclasts, and then TGF- $\beta$ from the resorbed bone causes EMT in the tumour cells.

In conclusion, our results indicate that cancer-derived PTHrP plays a critical role in OSCC mandibular bone invasion mediated by osteoclasts. The results of this study may provide a new approach for the treatment and management of OSCC.

\section{Acknowledgements}

This work was supported in part by a Grant-in-Aid for Scientific Research on Priority Areas 'Cancer' and for the 21st Century Center of Excellence program from the Ministry of Education, Culture, Sports, Science and Technology (MEXT) of Japan, Third Term Comprehensive 10-Year Strategy for Cancer Control from the Ministry of Health and Welfare of Japan to M.S., and Grant-in-Aid for Young Scientific Research on Priority Areas 'Cancer' from MEXT of Japan to T.M. We would like to express our sincere thanks to Dr Kouichi Matsuo for support throughout this work; Dr Toru Kiyono (Virology Division, National Cancer Center Research Institute) for providing the pENTR221 and pDESTSI-CMSCVpuro vectors; Shigeru Okano for $\mu$-CT technical assistance; and H. Suzuki, H. Abe, S. Kusakari, M. Fujiwara, M. Morioka, and M. Konno for providing expert technical assistance.

\section{References}

1. Barnes L, Eveson JW, Reichart P and Sidransky D: World Health Organization classification of tumours: pathology and genetics of head and neck tumours. IARC Press, Lyon, 2005.

2. Wong RJ, Keel SB, Glynn RJ and Varvares MA: Histological pattern of mandibular invasion by oral squamous cell carcinoma. Laryngoscope 110: 65-72, 2000.

3. Semba I, Matsuuchi $\mathrm{H}$ and Miura Y: Histomorphometric analysis of osteoclastic resorption in bone directly invaded by gingival squamous cell carcinoma. J Oral Pathol Med 25: 429-435, 1996.

4. Hicks WL Jr, Loree TR, Garcia RI, Maamoun S, Marshall D, Orner JB, Bakamjian VY and Shedd DP: Squamous cell carcinoma of the floor of mouth: a 20-year review. Head Neck 19: 400-405, 1997.

5. Brown JS, Lowe D, Kalavrezos N, D'Souza J, Magennis P and Woolgar J: Patterns of invasion and routes of tumor entry into the mandible by oral squamous cell carcinoma. Head Neck 24: 370-383, 2002. 
6. Nomura T, Shibahara T, Katakura A, Matsubara S and Takano N: Establishment of a murine model of bone invasion by oral squamous cell carcinoma. Oral Oncol 43: 257-262, 2007.

7. Krosl G, Korbelik M and Dougherty GJ: Induction of immune cell infiltration into murine SCCVII tumour by photofrin-based photodynamic therapy. Br J Cancer 71: 549-555, 1995.

8. O'Malley BW Jr, Cope KA, Johnson CS and Schwartz MR: A new immunocompetent murine model for oral cancer. Arch Otolaryngol Head Neck Surg 123: 20-24, 1997.

9. Guise TA, Yin JJ, Thomas RJ, Dallas M, Cui Y and Gillespie MT: Parathyroid hormone-related protein (PTHrP)-(1-139) isoform is efficiently secreted in vitro and enhances breast cancer metastasis to bone in vivo. Bone 30: 670-676, 2002.

10. Ohshiba T, Miyaura C, Inada M and Ito A: Role of RANKLinduced osteoclast formation and MMP-dependent matrix degradation in bone destruction by breast cancer metastasis. Br J Cancer 88: 1318-1326, 2003.

11. Lin DL, Tarnowski CP, Zhang J, Dai J, Rohn E, Patel AH, Morris MD and Keller ET: Bone metastatic LNCaP-derivative C4-2B prostate cancer cell line mineralizes in vitro. Prostate 47: 212-221, 2001

12. Yonou H, Ogawa Y and Ochiai A: Mechanism of osteoblastic bone metastasis of prostate cancer. Clin Calcium 16: 557-564, 2006 .

13. Yaccoby S, Wezeman MJ, Henderson A, Cottler-Fox M, Yi Q, Barlogie B and Epstein J: Cancer and the microenvironment: myeloma-osteoclast interactions as a model. Cancer Res 64: 2016-2023, 2004.

14. Roodman GD: Mechanisms of bone resorption in myeloma. J Musculoskelet Neuronal Interact 3: 271-272, 2003.

15. Guise TA, Yin JJ, Taylor SD, Kumagai Y, Dallas M, Boyce BF Yoneda T and Mundy GR: Evidence for a causal role of parathyroid hormone-related protein in the pathogenesis of human breast cancer-mediated osteolysis. J Clin Invest 98: 1544-1549, 1996

16. Shen X and Falzon M: PTH-related protein modulates PC-3 prostate cancer cell adhesion and integrin subunit profile. Mol Cell Endocrinol 199: 165-177, 2003.

17. Inoue J, Gohda J, Akiyama T and Semba K: NF-kappaB activation in development and progression of cancer. Cancer Sci 98: 268-274, 2007.
18. Roodman GD: Mechanisms of bone metastasis. N Engl J Med 350: 1655-1664, 2004.

19. Yin JJ, Selander K, Chirgwin JM, Dallas M, Grubbs BG, Wieser R, Massague J, Mundy GR and Guise TA: TGF-beta signaling blockade inhibits PTHrP secretion by breast cancer cells and bone metastases development. J Clin Invest 103: 197-206, 1999.

20. Nomura T, Shibahara T, Cui NH and Noma H: Patterns of mandibular invasion by gingival squamous cell carcinoma. J Oral Maxillofac Surg 63: 1489-1493, 2005.

21. Shibahara T, Nomura T, Cui NH and Noma H: A study of osteoclast-related cytokines in mandibular invasion by squamous cell carcinoma. Int J Oral Maxillofac Surg 34: 789-793, 2005.

22. Mori T, Kiyono T, Imabayashi H, Takeda Y, Tsuchiya K, Miyoshi S, Makino H, Matsumoto K, Saito H, Ogawa S, Sakamoto M, Hata J and Umezawa A: Combination of hTERT and bmi-1, E6, or E7 induces prolongation of the life span of bone marrow stromal cells from an elderly donor without affecting their neurogenic potential. Mol Cell Biol 25: 51835195,2005 .

23. Jungert K, Buck A, von Wichert G, Adler G, Konig A, Buchholz M, Gress TM and Ellenrieder V: Sp1 is required for transforming growth factor-beta-induced mesenchymal transition and migration in pancreatic cancer cells. Cancer Res 67: 1563-1570, 2007.

24. Karaplis AC and Goltzman D: PTH and PTHrP effects on the skeleton. Rev Endocr Metab Disord 1: 331-341, 2000.

25. Buchs N, Manen D, Bonjour JP and Rizzoli R: Calcium stimulates parathyroid hormone-related protein production in Leydig tumor cells through a putative cation-sensing mechanism. Eur J Endocrinol 142: 500-505, 2000.

26. Tarin D, Thompson EW and Newgreen DF: The fallacy of epithelial mesenchymal transition in neoplasia. Cancer Res 65: 5996-6000, 2005.

27. Thiery JP and Sleeman JP: Complex networks orchestrate epithelial-mesenchymal transitions. Nat Rev Mol Cell Biol 7: 131-142, 2006.

28. Thiery JP: Epithelial-mesenchymal transitions in tumour progression. Nat Rev Cancer 2: 442-454, 2002.

29. Zavadil J and Bottinger EP: TGF-beta and epithelial-tomesenchymal transitions. Oncogene 24: 5764-5774, 2005. 